\title{
In situ measurement of contact area in coated surfaces
}

\author{
J.-H. Sick \& G.-P. Ostermeyer \\ Institute of Dynamics and Vibrations, \\ Technical University of Braunschweig, Germany
}

\begin{abstract}
Few attempts to directly measure the contact area of solid bodies have been made. In fact, it shapes up as a complex problem, because there usually is no direct access to the relevant surface of the experimental setup. Promising work in electrical measurement of the contact area by relating it to the contact resistance has been made by Bowden, Tabor and Holm from the 1930s on. This method has some restrictions due to the mutability of contamination layers involved in the most sensitive part of the setup. It is suggested to reintroduce observation of the contact area by electrical measurement, taking advantage of recent progress made in thin film coatings. By use of low conductive coating materials like oxides or carbon based films the contact resistance is increased by several orders of magnitude. The measured value of contact resistance in low conductive coated samples is within a range to be measured nearly independent of parasitic inductions like usual contaminations. Current work is on quasi-statical experiments and calibration. The first results joining earlier efforts are reported and the experimental setup, preparation method, advantages and known limitations are put up for discussion.

Keywords: real contact area, contact model, friction, wear, contact resistance.
\end{abstract}

\section{Introduction}

The question of how to measure the real contact area of solid bodies directly leads to the problem of defining the real contact area. Relationship of load and electrical contact resistance is investigated, thus observing effects influenced by parameters on a subatomic scale, leading to results perceivable in macroscopic dimensions. To define what to address as the real contact area is not quite simple. In the experimental setup the contact of a solid body dipped into a conductive 
liquid is used to aim for a reproducible holohedral electric contact. This standard of comparison has several imperfections like the dependence to wettability of the surface, roughness, pressure, atmospheric composition etc. Nevertheless it can be useful to compare the immersion standard measurements with results from solid body contacts.

The contacts investigated by Bowden and Tabor were cleaned metal surfaces, for instance silver, steel, brass. Measured contact resistances vary from about $100 \cdot 10^{-6} \Omega$ for a load of $5 \mathrm{~N}$ and $50 \cdot 10^{-6} \Omega$ for a load of $5 \mathrm{kN} \mathrm{[3].} \mathrm{The} \mathrm{setup} \mathrm{of}$ Bowden and Tabor involves several precautions to obtain reliable results. This includes a cleaning procedure, four wire connection because of the contact resistance being low compared to the leads, and gentle movement of the surfaces before settling of the measured value. Results show sensitivity to the presence of contamination layers of any kind, as well.

Today one may choose from several facilities to prepare surfaces with well researched thin film coatings, raising the contact resistance by several orders of magnitude, providing a considerable improvement to the usability of this technique. The coating itself might change the character of the surface drastically, as found in tribological, rather nearly all physical and chemical properties. Nevertheless, a better insight to friction dynamics of tribological boundary layers may be anticipated. In micro hardness testing with nano indenters, used for thin film coatings, it is difficult to measure the mechanical properties of the coating itself and not the substrate. The all over mechanical behaviour of several coatings is within given limits similar to a flexible skin, that permits and follows deformations of the substrate before delamination happens. Even though the coating might be harder than the substrate itself, the macroscopic load carrying capacity of the composite is not increased, unless edge layer of the substrate is strongly biased like in nitrided or kolsterised steel (cf. Bach [2] et al).

\section{Experimental setup}

\subsection{Equipment}

For electrical measurement a low noise current source Keithley 6221 is used in combination with a Nanovoltmeter Keithley 2182A and a digital transient recorder Yokogawa DL750 with 16 bit resolution inputs. The Nanovoltmeter has an input biasing current $<5 \mathrm{nA}$ and an input resistance $>10 \mathrm{G} \Omega$ to be suitable for signal source resistances up to some Megaohm. For comparison of bandwidth, noise and load affects a Microdul HIP101 high impedance probe is used. This is configured as an input buffer with an input current of typical $0.2 \mathrm{pA}$ (FET leakage current at $293 \mathrm{~K}$ ), what is less than about $4 \mathrm{ppm}$ of the supply current used. All measurements are carried out in DC mode at low currents to achieve ohmic proportionality of voltage and current density. The motion speed is kept at low rates with regard to the bandwidth and resolution performance of the measurement instrumentation. Temperature is controlled with a drift of less than $\pm 0.1 \mathrm{~K}$ per hour. 
The probing force transducer is a Kistler type 9207, connected to a charge amplifier type 5019B140. A small drift typical to this device is compensated calculational retrospectively to data acquisition.

To measure displacement of the sample holder a Micro-Epsilon laser position encoder LD1605-20 is used.

Resistances in the measuring chain are small for all wiring, substrates, immersion, constriction resistance and current source resistance with the exception of the film resistance, boosting the contact resistance, and even more for the instrument input stages. For quasi-statical constitutional changes as in this case noise is kept at a low level by low pass filtering with a cut-off frequency of $10 \mathrm{~Hz}$ in Nanovoltmeter Voltage measurement, $100 \mathrm{~Hz}$ in force measurement, $400 \mathrm{~Hz}$ acquisition bandwidth limit and post acquisition cut-off to $10 \mathrm{~Hz}$ in high impedance probe voltage measurement (phase-neutral filter).

\subsection{Preparation}

Sample substrates are made of common stainless steel EN 1.4301/ASTM 304, polished sheet metal. With a Brinell hardness of about 170 and a Young's modulus of 200 Gigapascal this material grade is good machineable with the benefit of good corrosion resistance, what eases handling and storage of samples. Coated samples have a carbon based coating of $4 \mu \mathrm{m}$ thickness, available as diamond like carbon (DLC) for anti-wear purposes in machine parts. For flat-ball contact bearing balls made of EN 1.3505/ASTM A-295 are used, which is remarkably harder than the coated substrate, as well as the coating is. It is important to compare samples from the same coating lot each, because obtained thickness and conductance may scatter significantly. Samples are kept untouched after coating, stored in an exsiccator, contaminated with oxygen and moisture just due to exposition whilst handled in the laboratory.

\subsection{Contact area standard of comparison}

To align measured values of contact resistance to a known relation of resistance to contact area there is need for a reproducible standard to refer to. This involves definition of the contact area as itself to be able to give some absolute values. At present this definition appears to be only partly finished by metrologic institutes. In the first instance a standard experiment is used with a simple, practical definition of the contact area. The sample is immersed into mercury, which is liquid at lab temperature of $294 \mathrm{~K}$. The electrical resistivity of mercury is $95.9 \cdot 10^{-8} \Omega \mathrm{m}$ at $294 \mathrm{~K}$ (calculated from the CRC Handbook of Chemistry and Physics [5]), what is a high value for a metal (mercury is a relative weak conductor). The transition metals iron, nickel, chromium, manganese are hardly soluble in mercury, they do not form intermetallic phases with mercury as in amalgames like silver and copper in mercury. No change in the properties of the steel substrate material as well as the carbon film is to be due to contact with mercury, over and above the solid steel is not wettable to mercury in general. Aluminium for example amalgamates, but is usually not wettable due to its tight mercury-repellent oxide layer, investigated by [13]. 
In the experiment a coated electrode sheet is attached to an electrical insulating holder. By lowering the holder with a remote controlled, vibration-free motor drive the electrode is immersed to and extricated from a bath of mercury (Fluka, Hg puriss.). The mercury is kept in a glass vessel and is attached by a set of two wire electrodes of stainless steel. A schematic representation of the setup is given in Figure 1. The setup is built onto a vibration-isolated table to keep the mercury surface even. The level of the holder is measured by a laser triangulometer synchronously. Change in the mercury level due to displacement caused by the dipped electrodes is compensated upon data interpretation.

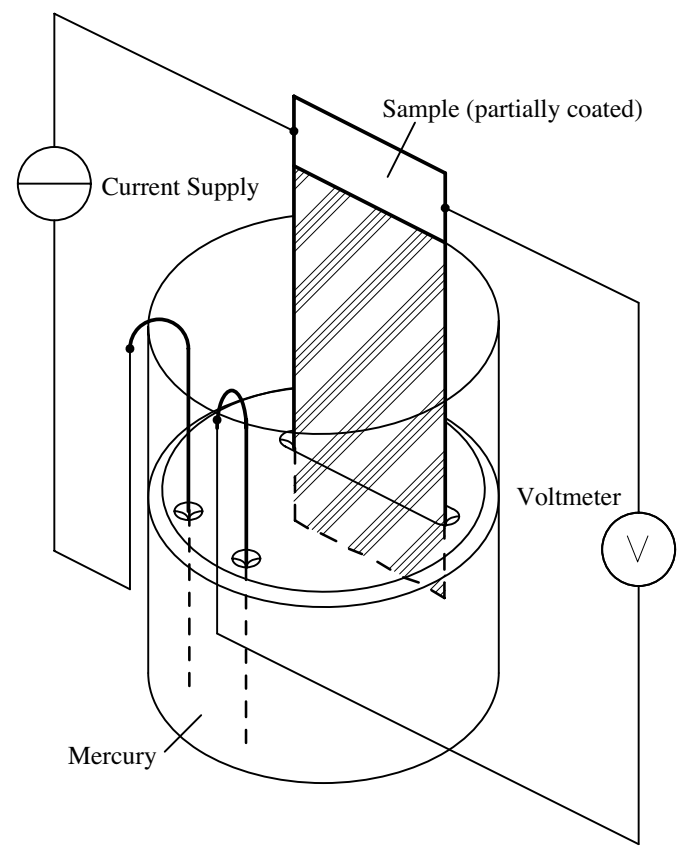

Figure 1: Schematic representation of the coated sample immersed into mercury to obtain holohedral contact.

\subsection{Static loaded solid-solid contacts}

Aim of the investigation is to obtain data about the real contact area depending on acting load by monitoring the contact resistance. The concept of "contact resistance" shall be used in terms of Holm, i.e. a resistance at the conduction area of the contact, no matter if there is a pure constriction resistance at a clean surface or a film contributing to the resistance [9].

To allow precision measurement, quasi-statical load-unload cycles are performed without lateral relative movement of the samples. The coated flat sample is mounted upside down to a perpendicularly driven rack. It is the same holder as used for the immersion experiment. The ball with a diameter of $10 \mathrm{~mm}$ is held in a countersink coaxial on the tip of a force transducer. The assembly 
compensates angular misalignment and keeps any electrical junctions symmetrical, except for the contact under test. The wires are clamped directly to the ball and the flat sample respectively, supply and measuring wires separately (4 wire connection). A schematic representation of the setup is given in Figure 2 (ball on flat sample). Current is kept constant at $50 \mathrm{nA}$, what turns out to be a value suiting well the ranges of the involved supply and meters, avoiding unwanted heating of the contact. Voltage measurement is accomplished in a fixed range of $12 \mathrm{~V}$ for the Nanovoltmeter and $9 \mathrm{~V}$ for the high impedance probe. Load effects are small for the Nanovoltmeter and negligible for the high impedance probe. They achieve results close to each other. The supply voltage is limited to $12 \mathrm{~V}$, thus lowering electrical power loss to a maximum of $0.12 \mu \mathrm{W}$. The integration time is set to 1 power line cycle $(20 \mathrm{~ms})$ for suppression of high frequency noise and mains hum.

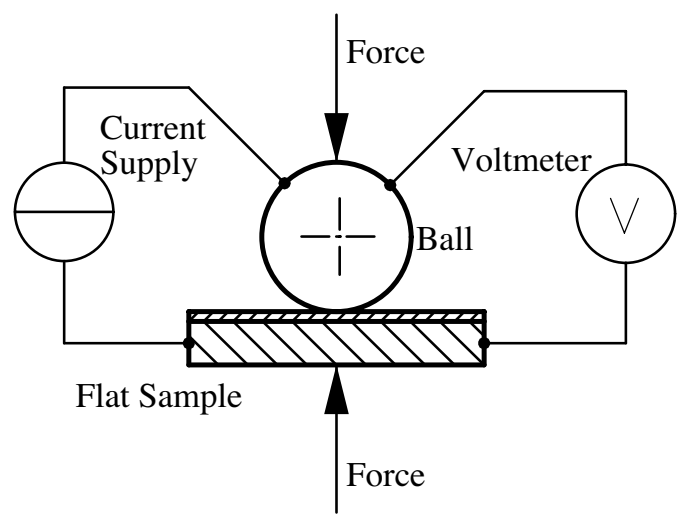

Figure 2: $\quad$ Schematic representation of the ball on flat contact.

\subsection{Known issues}

\subsubsection{Temperature}

Electric conductivity is temperature dependent. In case of metals like mercury and iron we have a small positive temperature coefficient of resistance (TCR). Semiconductive carbon based films show a strong temperature dependency. Robertson reports carbon films with a negative TCR in the range of $1 \%$ per Kelvin at $294 \mathrm{~K}$ [12]. Even small temperature changes or gradients within the sample will have a noticeable influence to the conductivity. The contact angle of mercury to several substrates is reported to be temperature dependent, as accounted from Ellison et al [7].

To avoid relevant uncertainties of temperature all experiments are arranged in a full-time thermocontrolled laboratory at a temperature of about $294 \mathrm{~K}$, performed one day of settling time after each handling of samples.

\subsubsection{Capacitance}

High contact resistances may have a noticeable capacitance, thus producing a delay in voltage changes due to current control. They might be part of the cause for a hysteresis found in several measurements. 


\subsubsection{Square resistance}

The lateral electric conduction will cause an additional non-linearity to the ideally reciprocal dependence of immersion depth and contact resistance. The effect decreases for a rising contact area.

\subsubsection{Semiconductive properties}

Robertson reports carbon based films as semiconductors with a typical dependency of current density and resistance [12]. Thus the current has to be kept very low or the voltage across the junction has to be kept constant, to avoid a non-linear response to changes of junction's cross sectional area that is involved in transport. Some films have piezoresistive properties, as shown from Tibrewala [14]. The polarity dependence found in measured results is small.

\subsubsection{Wettability, adhesion}

According to de Wet, there is no dissolving of mercury to the steel sample itself to be expected [16]. Wright reports little adherence of mercury to steel [17] and Cuthrell even less to carbon [6], if not particularly plasma treated as reported from Sudarshan et al [13].

A common method to measure the open porosity of solids non-wettable by mercury is known as mercury porosimetry. Mercury porosimetry characterises a material's porosity by applying various levels of pressure to a sample immersed in mercury. The pressure required to intrude mercury into the sample's pores is inversely proportional to the size of the pores, as described at Micromeritics [11]. Thus a pressure dependency in contact resistance of rough surfaces is most likely. Probably a computational correction for hydrostatic pressure should be applied. This is to be investigated separately.

\subsubsection{Contamination layers}

Both sides of the contact, solid and liquid, may be contaminated with layers of oxides and adsorbed moisture. These layers affect the contact directly by adding an accessory resistance as well as indirectly by altering the wettability, the latter reported from Sudarshan et al [13]. The accessory resistance of thin contamination layers themselves is very small in relation to the resistive coating, but relevant on blank surfaces, as Holm found already [9].

\subsubsection{Thermoelectric forces}

There will be a typical thermoelectrical force induced voltage at any junction in the measurement chain. The setup is elaborated to provide symmetry of electrical junctions, thus compensating for thermoelectric forces (except for the contact under test itself). Measuring current is kept at low values to avoid heating of the samples. As long as the temperature of antipodal junctions is kept equal they will add just some noise to the result (Low Level Measurement Handbook [10]).

For the coated samples measured voltages are up to several volts, so the influence of thermoelectric forces in the range of millivolts may be neglected. 


\subsubsection{Reference sensors}

Sensors used as reference for load and displacement have certain non-linearity and hysteresis, adding up to a relevant measurement uncertainty of up to $1.1 \%$ of the particular range.

\section{Results}

\subsection{Immersion of coated sample in mercury}

To measure electric properties of thin films they usually are deposited on a nonconductive substrate, hence electrical measurement proceeds with lateral direction of carrier stream in the film. In the present case the setup for measurement of electric conductivity shall be at the best accordance to the solid body contact to be compared with. The metal mercury, molten at room temperature, permits to make a setup with carrier stream normal to the film, resembling a lot of the electronic properties of the solid body contact.

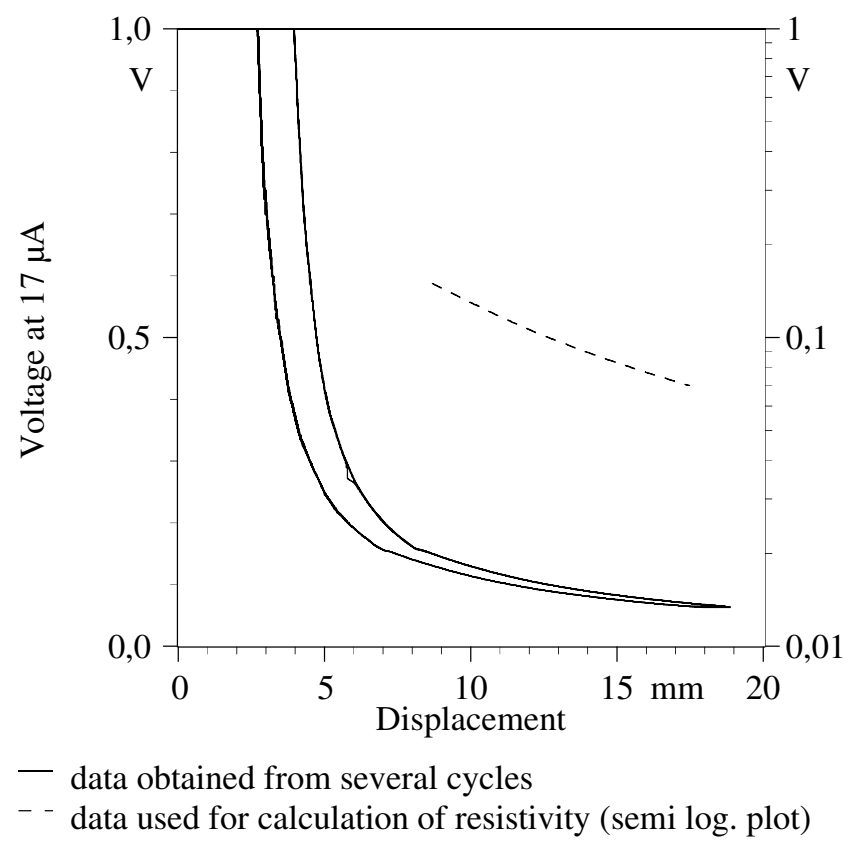

Figure 3: $\quad$ Measured voltage vs. vertical displacement of sample.

The conductivity calculated from the obtained data is about $3.5 \cdot 10^{-4} \Omega^{-1} \mathrm{~m}^{-1}$, what is within a possible range as reported from Robertson, Holiday $[12,8]$. The specific electrical resistance is well within typical ranges for various amorphous carbon films, as portrayed in VDI guideline 2840 [15]. 


\subsection{Flat-ball contact under normal load}

The contacts are tested in normal load cycles without lateral movement from 0 $10 \mathrm{~N}$ up-and-down.

For comparison the contact of a bearing ball and a blank metal sheet is investigated, too. Low voltages in the millivolt range have to be measured to keep the current low. A current of $50 \mu \mathrm{A}$ turns out to be suitable for the given contact to measure within a range of $10 \mathrm{mV}$ (except for the first few Micronewton of load, what is beyond access due to a voltage compliance to limit the electrical power loss). This supply current is a thousand times the current used for the resistive coated samples. The result is shown in Figure 4 and 5 in plots of voltage and conductance vs. load. Note the very distinctive hysteresis with higher voltage for increasing load, which was found similar in several runs.

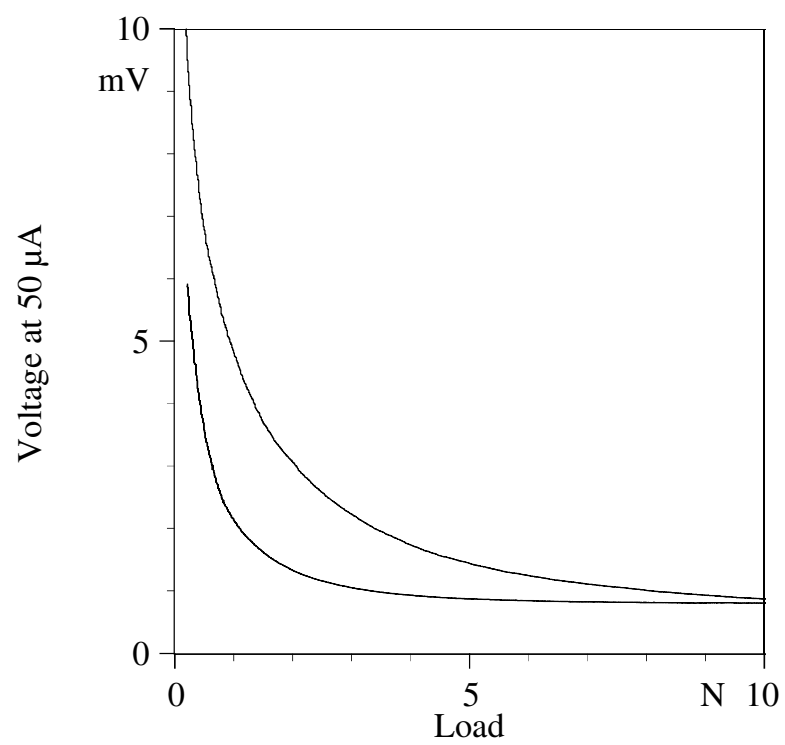

Figure 4: $\quad$ Measured voltage vs. load for blank ball on flat contact.

A resistive coated sample of the same substrate is contacted by a blank bearing ball. Due to the raised resistance the measured voltage is within a plainly gaugeable range of $10 \mathrm{~V}$ for a current as small as $50 \mathrm{nA}$. To afford measurement on a high impedance signal source like this contact an instrumentation amplifier with a field effect transistor input stage is useful, otherwise the electrical load on the DUT might corrupt the result.

Results measured with a high impedance probe are shown in Figure 6. From the measured voltage the conductance is calculated to produce the common logarithmical plot of conductance vs. load given in Figure 7.

Combined with the area resistance proportion from the immersion experiment the conductance is plotted as real contact area vs. load in Figure 8. 
Computer Methods and Experimental Measurements VIII 267

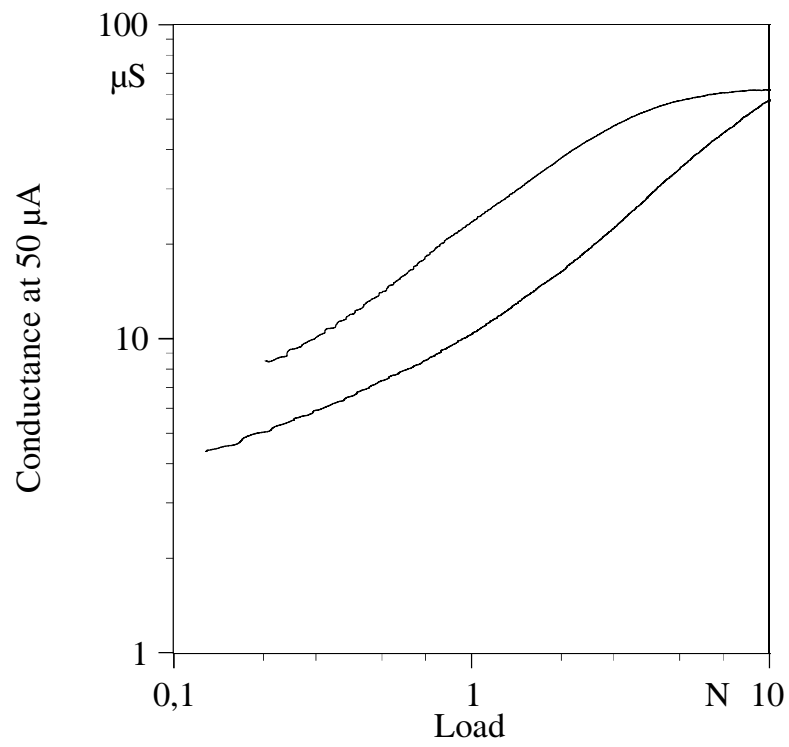

Figure 5: Calculated conductance vs. load for blank ball on flat sample.

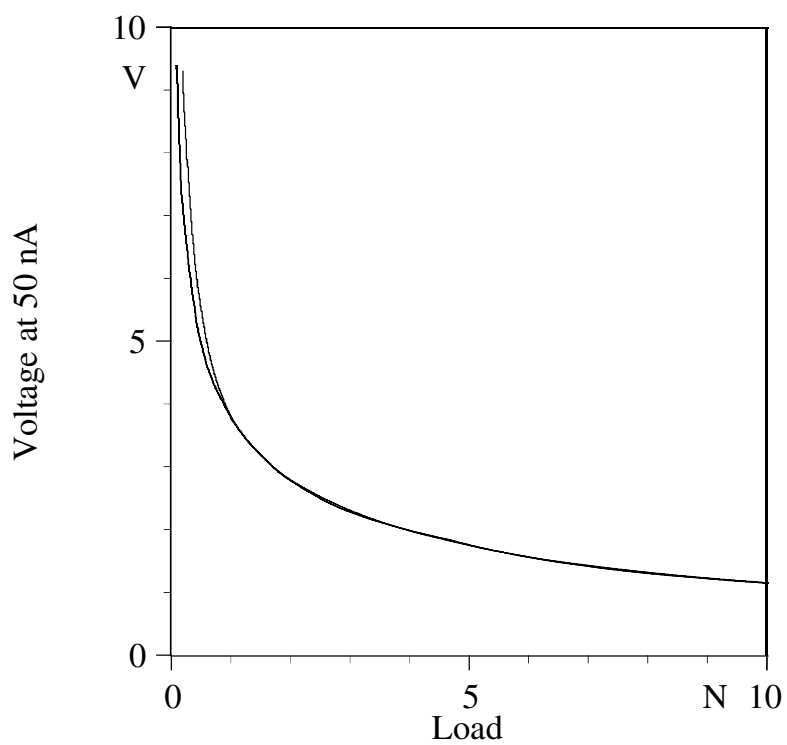

Figure 6: $\quad$ Measured voltage vs. load for ball on coated flat sample. 
268 Computer Methods and Experimental Measurements VIII

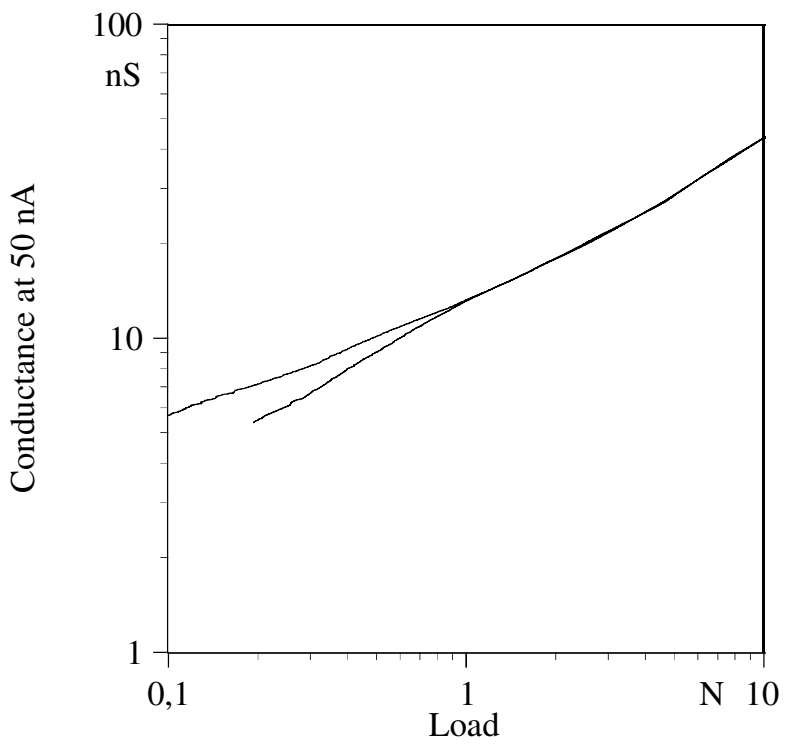

Figure 7: Calculated conductance vs. load for ball on coated flat sample.

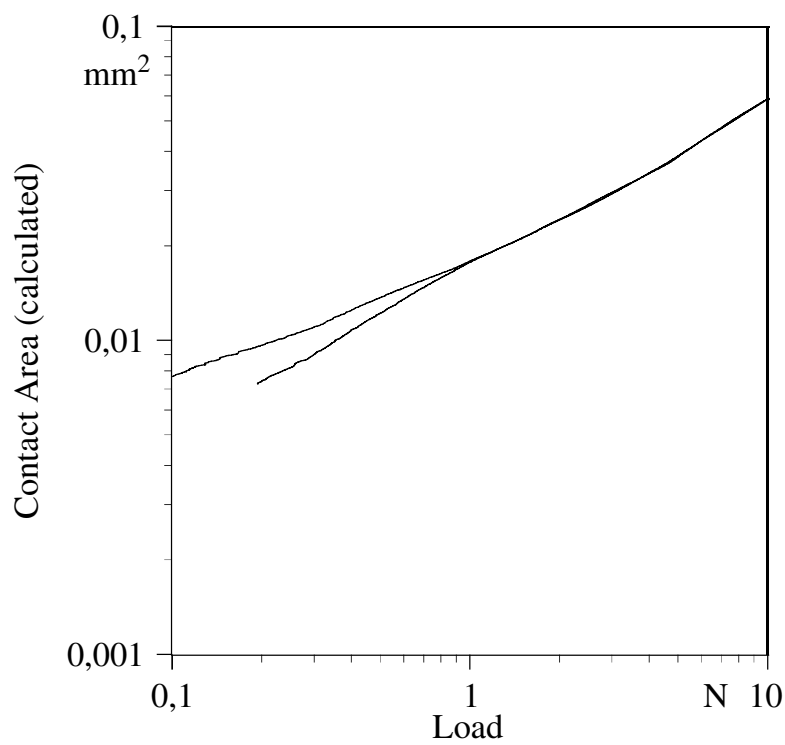

Figure 8: Calculated contact area vs. load for ball on coated flat sample.

Note the linear dependence of load and contact area. This is similar to recent predictions based on numerical calculations from Almqvist as well as from Ciavarella $[1,4]$. 


\section{Discussion and conclusions}

It is shown that a configuration of a resistive coated steel sample and a blank counterpart permits convenient measurement of contact resistances. The dependence of measured conductance to load is almost linear already for small load, as expected from theory. The contact area is quantified for the given setup. Several issues mentioned above might cause a non-ideal contact load response of electric contact properties. Each of this will be subject to further investigations.

An alternative to the proposed immersion method with isostatic solid-liquid contact would be to build solid conductive electrodes, either made of electrical conductive gluing compound or of vapour deposited metal films (as used in preparation of samples for electron microscopy). Such electrodes on top of the resistive layer should obtain full tangency on a measurable portion of the surface. It might result some metal doping of the resistive layer however, leading to a resistivity different from the not treated area. Accurate measurement of conductivity and thickness (using known relationship of film thickness, specific resistance and square resistance as well as eddy current testing and profilometry), and investigation of anisotropic features of individual coatings could help to adjust another reference value for the relation of contact area and contact resistance.

\section{Acknowledgement}

This work was supported by the German Research Foundation (DFG), under contract number OS 166/2-1.

\section{References}

[1] Almqvist, A., Sahlin, F., Larsson, R., Glavatskih, S., On the dry elastoplastic contact of nominally flat surfaces, Tribology International 40, 574 579, 2007.

[2] Bach, F.-W., Moehwald, K., Laarmann, A., Wenz, T. (editors), Moderne Beschichtungsverfahren, Wiley-VCH, Weinheim, 2004.

[3] Bowden, F. P., Tabor, D., Reibung und Schmierung fester Koerper. Springer-Verlag, Berlin, 32, 1959.

[4] Ciavarella, M., Delfine, V., Demelio, G., A "re-vitalized" Greenwood and Williamson model of elastic contact between fractal surfaces, Journal of the Mechanics and Physics of Solids 54, Elsevier, 2569-2591, 2006.

[5] CRC Handbook of Chemistry and Physics, Chemical Rubber Publishing Company, New York, 79th edition 1998-1999.

[6] Cuthrell, R. E., Evaluation of electrical contact materials for mercury switches designed to detect angular rotation, Journal of Materials Science, Chapman and Hall Ltd., 21, 2119-2123, 1986.

[7] Ellison, A. H., Klemm, R. D., Schwartz, A. M., Grubb, L. S., and Petrash, D. A., "Contact angles of mercury on various surfaces and the effect of temperature," J. Chem. Eng. Data 12, No. 4, 607, 1967. 
[8] Holiday, P., Dehbi-Alaoui, A., Matthews, A., Physical properties of carbon films produced using a hybrid vapour deposition technique, Surface and Coatings Technology 47, Elsevier, 315-326, 1991.

[9] Holm, R., Electric Contacts, 4th edition, Springer-Verlag, Berlin, 1967.

[10] Low Level Measurements Handbook, 6th Edition, Keithley Instruments Inc., Cleveland, Ohio, 2004.

[11] Mercury Porosimetry Using AutoPore Porisimeters From Micromeritics published on AZoM at http://www.azom.com/details.asp?ArticleID=3227

[12] Robertson, J., Diamond-like amorphous carbon, Materials Science and Engineering, Elsevier Science B.V., R 37, 236, 242, 2002.

[13] Sudarshan, T. S., Lim, M. H., Hefley, P. L., Thompson, J. E., Wetting of aluminium electrodes with mercury, Journal of Applied Physics, American Institute of Physics, 56 (8), 2236-2240, 1984.

[14] Tibrewala, A., Piezoresistive effect in diamond-like carbon films (dissertation, Technical University of Braunschweig, Germany), Cuvillier Verlag, Goettingen, 2006.

[15] VDI Verein Deutscher Ingenieure (The Association of Engineers) (Editor), guideline VDI 2840 "Carbon coatings - Basic knowledge, coating types and properties", Beuth Verlag GmbH, Berlin, Germany, 2005.

[16] de Wet, J. F., Haul, R. A. W., Zur Loeslichkeit einiger Uebergangsmetalle in Quecksilber, Zeitschrift fuer anorganische und allgemeine Chemie, WILEY-VCH Verlag GmbH \& Co. KGaA, Weinheim, Volume 277, Issue 12, 96-112, 1954.

[17] Wright, D. J., Hysteresis of the Angle of Contact of Mercury against Steel, Proc. Phys. Soc. B 68 297-303, 1955. 